TITLE:

\title{
Model of a switching oxide trap in amorphous silicon dioxide
}

AUTHOR(S):

Uchino, T; Takahashi, A; Yoko, T

\section{CITATION:}

Uchino, $T$... [et al]. Model of a switching oxide trap in amorphous silicon dioxide. PHYSICAL REVIEW B 2001, 64(8): 081310.

ISSUE DATE:

2001-08-15

URL:

http://hdl.handle.net/2433/50389

\section{RIGHT:}

Copyright 2001 American Physical Society 
PHYSICAL REVIEW B, VOLUME 64, 081310(R)

\title{
Model of a switching oxide trap in amorphous silicon dioxide
}

\author{
T. Uchino, ${ }^{*}$ M. Takahashi, and T. Yoko \\ Institute for Chemical Research, Kyoto University, Uji, Kyoto 611-0011, Japan
}

(Received 12 June 2001; published 8 August 2001)

\begin{abstract}
We present a model of a switching oxide trap in amorphous silicon dioxide on the basis of quantum chemical calculations on clusters of atoms. We show that the positively-charged defect center proposed in previous papers [Uchino et al., Phys. Rev. B 62, 2983 (2000); Phys. Rev. Lett. 86, 5522 (2001)] can capture an electron without accompanying complex atomic rearrangements, forming a metastable hole-electron pair that can in turn emit an electron. The present model also gives a reasonable account for the cathodoluminescence and thermally stimulated luminescence emissions at $445 \mathrm{~nm}$ from amorphous silicon dioxide.
\end{abstract}

DOI: 10.1103/PhysRevB.64.081310

PACS number(s): 61.72.Ji, 61.43.Fs, 68.35.Dv

Amorphous silicon dioxide $\left(a-\mathrm{SiO}_{2}\right)$ is the most widelyused thin-film insulator in modern microelectronic devices, including a gate or field dielectric for metal-oxidesemiconductor (MOS) devices. Since the performance and reliability of the amorphous insulator strongly depends on the presence of charge trapping centers, charge trapping in $a-\mathrm{SiO}_{2}$ has been the subject of intensive study during the past decades. ${ }^{1-4}$ It is well accepted that charge trapping in amorphous insulators results from microstructural defect centers. In particular, there have been considerable experimental and theoretical studies concerning a switching defect in $a-\mathrm{SiO}_{2}$ since switching oxide traps can repeatedly "switch" charge states in response to changes in the voltage applied to the gate of a MOS field-effect transistor. ${ }^{5}$ Recently, combining electrical measurements and electron spin resonance (ESR) techniques, Conley et al. ${ }^{6}$ have demonstrated that the ESR signal associated with the $\mathbf{E}_{\gamma}^{\prime}$ centers grow and decay as a function of the sign of the electric field in $a-\mathrm{SiO}_{2}$. The conventional structural model of the $\mathbf{E}_{\gamma}^{\prime}$ center is a hole trapped at an oxygen monovacancy, $\equiv \mathrm{Si}^{+} \cdot \mathrm{Si} \equiv$, where $\equiv$ and $\cdot$ represent the three $\mathrm{Si}-\mathrm{O}$ bonds and the unpaired electron, respectively. The experimental results of Conley et al. ${ }^{6}$ clearly indicate that the $\mathbf{E}_{\gamma}^{\prime}$ centers can account for both hole traps and switching oxide traps; that is, no complex structural arrangements may occur at the hole trapping site ( $\mathbf{E}_{\gamma}^{\prime}$ centers) after subsequent electron capture.

Lelis and co-workers ${ }^{2,7}$ have previously developed a model for such a switching behavior of the $\mathbf{E}_{\gamma}^{\prime}$ center. They suggested that the switching was accomplished by capturing and emitting an electron from the dangling silicon (三 $\mathrm{Si} \cdot$ ) orbital without changing the electronic structure of the hole part of the defect $\left(\equiv \mathrm{Si}^{+}\right)$. However, their model has not been universally accepted because of the following reasons. ${ }^{8}$ Firstly, it is counterintuitive to assume that the electron would be captured on the paramagnetic part of the defect instead of the coulombically-attractive hole part. Secondly, even if the paramagnetic part captures an electron, the resultant doubly-occupied silicon dangling orbital would result in a high-energy metastable state, and, therefore, the hole $\left(\equiv \mathrm{Si}^{+}\right)$-electron $\left(\equiv \mathrm{Si}^{-}\right)$pair will be easy to recombine to form a stable $\mathrm{Si}-\mathrm{Si}$ bond that will not emit an electron. The energy barrier to the $\mathrm{Si}-\mathrm{Si}$ bond formation from such a metastable state has been evaluated theoretically. ${ }^{9,10}$ The cal- culated barrier has been shown to be so small $(\sim 0.2 \mathrm{eV})$ that the expected lifetime for the metastable state would be very short at room temperature and above; that is, the calculated barrier would be too small to explain the thermal stability of the switching oxide trap in $a-\mathrm{SiO}_{2} \cdot{ }^{8,11}$ Nevertheless, there is compelling experimental evidence that the $\mathbf{E}_{\gamma}^{\prime}$ center can repeatedly "switch" charge state with changes in gate bias, suggesting that rather a stable hole-electron pair is formed from the $\mathbf{E}_{\gamma}^{\prime}$ center.

This seemingly irreconcilable behavior concerning switching traps in $a-\mathrm{SiO}_{2}$ may result from the inappropriate structural model of the $\mathbf{E}_{\gamma}^{\prime}$ center, which was originally proposed by Feigle, Fowler, and Yip (FFY) to explain the experimental electron spin resonance (ESR) characteristics of the $\mathbf{E}_{1}^{\prime}$ center in $\alpha$-quartz. ${ }^{12}$ Indeed, the FFY model, although never verified experimentally, successfully explains the localization of the unpaired electron in the positively-charged defect center, which is one of the main characteristics seen in the ESR spectrum of the $\mathbf{E}_{\gamma}^{\prime}$ center. However, we have recently proposed an alternative structural model of the $\mathbf{E}_{\gamma}^{\prime}$ center that can also account for the localization of the unpaired spin on a single silicon atom. ${ }^{13}$ This model consists of two types of three-coordinated silicons. One is paramagnetic $(\equiv \mathrm{Si} \cdot)$ and the other positively-charged $\left(\equiv \mathrm{Si}^{+}\right)$, similar to the case of the FFY model; however, we assumed that these two types of silicon atoms can be bridged by a common oxygen atom, resulting in the following configuration: $=(\mathrm{Si} \cdot)-\mathrm{O}-\left(\mathrm{Si}^{+}\right)=$. We have demonstrated that this type of defect, termed a bridged hole-trapping oxygen-deficiency center (BHODC), quantitatively reproduces the large ${ }^{29} \mathrm{Si}$ hyperfine splitting $(\sim 42 \mathrm{mT})$ observed for the $\mathbf{E}_{\gamma}^{\prime}$ center. $\mathrm{We}^{13}$ have also demonstrated that one possible neutral precursor of BHODC is the defect in which one of the two oxygens in the edge-sharing structural unit is missing; this neutral defect center is called a triangular oxygen-deficiency center (TODC),

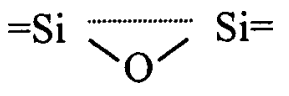

Stefanov et al. ${ }^{14}$ have recently reported that such a defect configuration as seen in TODC indeed exists at the $\mathrm{Si}-\mathrm{SiO}_{2}$ interface as an intermediate during silicon oxide formation. 


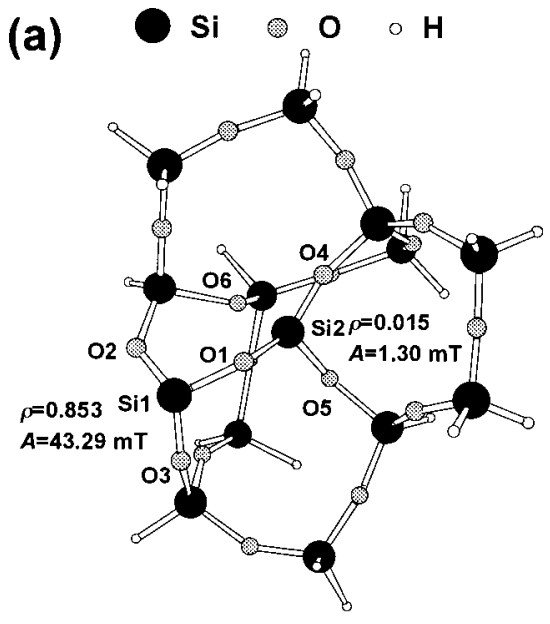

Bond distances (A) Si1-01=1.679 Si $1-02=1.680$ Si $1-03=1.627$ $\mathrm{Si} 2-01=1.620$ $\mathrm{Si} 2-04=1.603$ $\mathrm{Si2}-05=1.612$ $\mathrm{Si} 2-06=1.788$ $\mathrm{Si} 1-\mathrm{Si} 2=3.038$

Bond angles (degree) $\mathrm{Si} 1-01-\mathrm{Si} 2=134.0$ $01-\mathrm{Si} 1-02=102.0$ $01-\mathrm{Si1}-03=110.3$ O2-Si1-03=109.8 $01-\mathrm{Si} 2-04=111.6$ $01-\mathrm{Si} 2-05=114.8$ $\mathrm{O}-\mathrm{Si2}-\mathrm{O} 5=116.4$

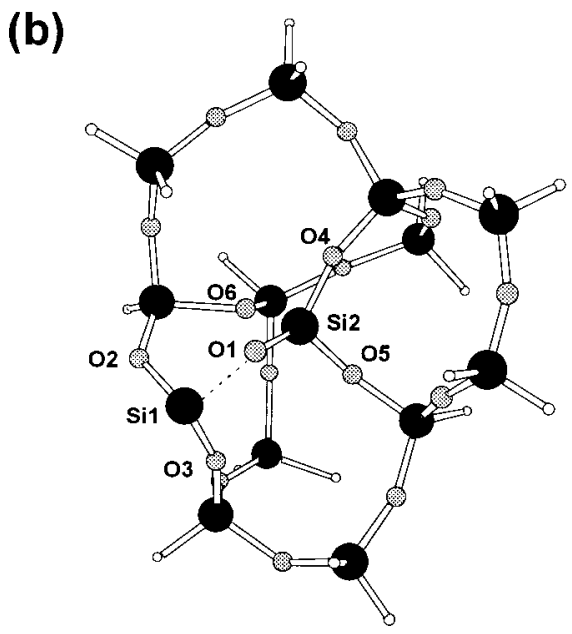

TABLE I. Mulliken atomic charges of defect centers in the present cluster models.

\begin{tabular}{lrrrr}
\hline \hline Atom & Model 1 & Model 2 & Model 3 & Model 4 \\
\hline Si1 & 1.055 & 0.749 & 0.795 & 0.638 \\
Si2 & 1.269 & 1.257 & 0.800 & 1.216 \\
O1 & -0.631 & -0.663 & -0.578 & -0.618 \\
\hline \hline
\end{tabular}

Fig. 1 that when an electron is added to BHODC, one of the $\mathrm{Si}-\mathrm{O}$ bonds in the defect site $(\mathrm{Si} 2-\mathrm{O} 1)$ becomes shorter, whereas the rest of the $\mathrm{Si}-\mathrm{O}$ bond $(\mathrm{Si} 1-\mathrm{O} 1)$ becomes longer. Table I shows the atomic charges $q$ of $\mathrm{Si}$ and $\mathrm{O}$ atoms in the respective defect sites. We see from Table I that $q_{\mathrm{Si} 2}$ in model $2\left(q_{\mathrm{Si} 2}=1.257\right)$ is almost comparable to the atomic charge of the hole center in model $1\left(q_{\mathrm{Si} 2}=1.269\right)$, showing the positive character of $\mathrm{Si} 2$ in model 2. It is also interesting to note that these hole-trapping silicon atoms appear to interact with a nearby oxygen atom $(\mathrm{O} 6)$; the resultant $\mathrm{Si} 2-\mathrm{O} 6$ interatomic distances in models 1 and 2 are calculated to be 1.788 and 1.793 , respectively. On the other hand, $q_{\mathrm{Si}}$ in model 2 $\left(q_{\mathrm{Sil}}=0.749\right)$ is even smaller than the corresponding atomic charge in model $1\left(q_{\mathrm{Sil}}=1.055\right)$. This indicates that the negative charge is located around Si1 in model 2. It can hence safely be said that in model 2 a hole and an electron are separately located in the defect; that is, a hole-electron pair is expected to be formed.

This hole-electron pair would be stable as long as it retains the coordination environment shown in Fig. 1(b). Stated in another way, rather a stable hole-electron pair will be formed from BHODC without accompanying complex structural rearrangements, preceded by electron capture at this positively-charged site. These calculated results allow us to suggest that BHODC does act as a switching trap; the

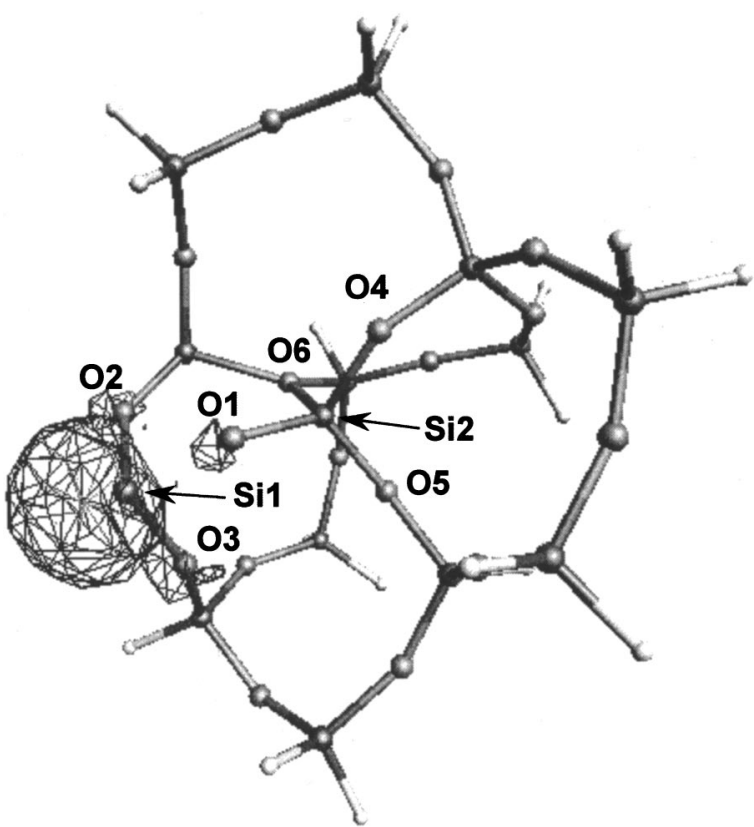

FIG. 2. A schematic view of the highest-occupied-molecularorbital calculated for model 2 . 


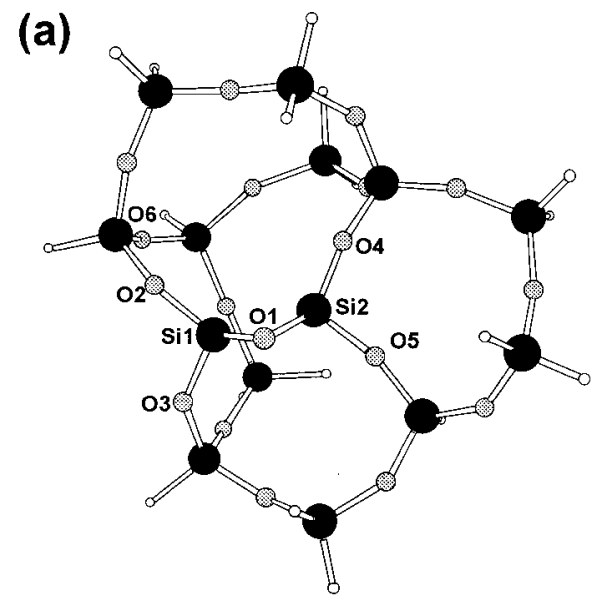

Bond distances $(A)$

Si1-01 $=1.700$

Si1-02 $=1.637$

Si $1-03=1.642$

$\mathrm{Si} 2-01=1.705$

$\mathrm{Si} 2-04=1.640$

$\mathrm{Si} 2-05=1.629$

$\mathrm{Si} 2-06=4.611$

$\mathrm{Si} 1-\mathrm{Si2}=\mathbf{2 . 1 8 3}$

Bond angles (degree)

$\mathrm{Si} 1-01-\mathrm{Si} 2=79.8$

O1-Si1-O2=115.0

O1-Si1-O3=115.0

$\mathrm{O} 2-\mathrm{Si} 1-\mathrm{O}=107.8$

01-Si2-04=113.0

01-Si2-05=115.3

O4-Si2-05=107.7

(b)

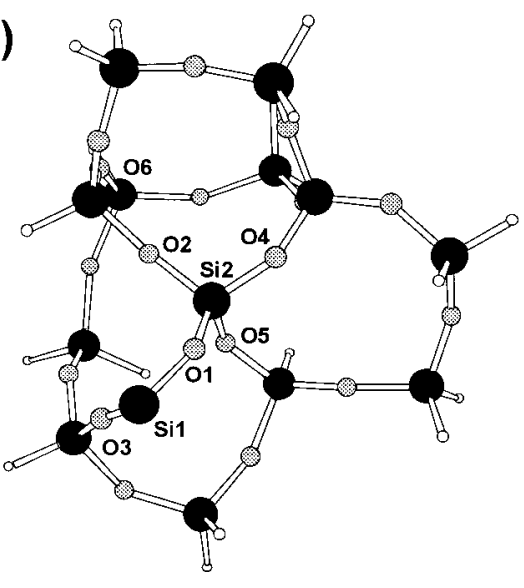

Bond distances (A)

Si1-01=1.676

Si1-03 $=1.668$

Si2-01=1.644

$\mathrm{Si2}-02=1.623$

$\mathrm{Si} 2-04=1.640$

$\mathrm{Si} 2-05=1.637$

$\mathrm{Si2}-06=4.077$

Si1-Si2 $=3.097$

Bond angles (degree)

Si1-01-Si2=137.9

O1-Si1-O3=102.7

$01-\mathrm{Si} 2-02=109.0$

O1-Si2-04=108.3

01-Si2-05=110.4

$\mathrm{O} 2-\mathrm{Si} 2-04=109.9$

$\mathrm{O} 2-\mathrm{Si} 2-\mathrm{O}=108.8$

O4-Si2-O5=110.3

FIG. 3. Lower energy configurations of the $\mathrm{Si}_{14} \mathrm{O}_{18} \mathrm{H}_{18}$ cluster optimized at the B3LYP/6-31G(d) level: (a) a triangular oxygendeficiency center (model 3); (b) a divalent Si defect (model 4). The total energies of model 3 and model 4 are lower than that of model 2 by 0.05 and $1.23 \mathrm{eV}$, respectively.

switching will be realized by capturing an electron at BHODC to form the hole-electron pair, which will in turn emit an electron to form BHODC.

It should also be noted that the highest occupied molecular orbital of model 2 is mostly characterized by $\mathrm{Si} 3 p$ atomic orbitals associated with a lone pair of electrons on Si1 (see Fig. 2) as in the case of the Si divalent defect. The atomic charge of Si1 in model $2\left(q_{\mathrm{Si1}}=0.749\right)$ is almost comparable to that of the Si divalent defect in model 4, which will be shown below $\left[q_{\mathrm{Si1}}=0.638\right.$, see also Fig. 3(b) $]$. Thus, Si1 in model 2 is expected to have almost the same electronic structure as that for the usual divalent Si defect. The formation of the divalentlike defect from TODC may account for the cathodoluminescence (CL) or thermally stimulated luminescence (TSL) emissions at $445 \mathrm{~nm}$ from $a-\mathrm{SiO}_{2} \cdot{ }^{18,19}$ Since the CL and TSL emissions result from the recombination process of a released electron with a positively-charged center, it has been proposed that the $\mathbf{E}_{\gamma}^{\prime}$ center is responsible for these emissions. ${ }^{18}$ It should also be worth mentioning that the CL and TSL emissions centered at $445 \mathrm{~nm}$ coincide with the triplet-to-singlet photoluminescence band associated with the divalent $\mathrm{Si}$ defect in $a-\mathrm{SiO}_{2} \cdot{ }^{20}$ In the CL and TSL pro-

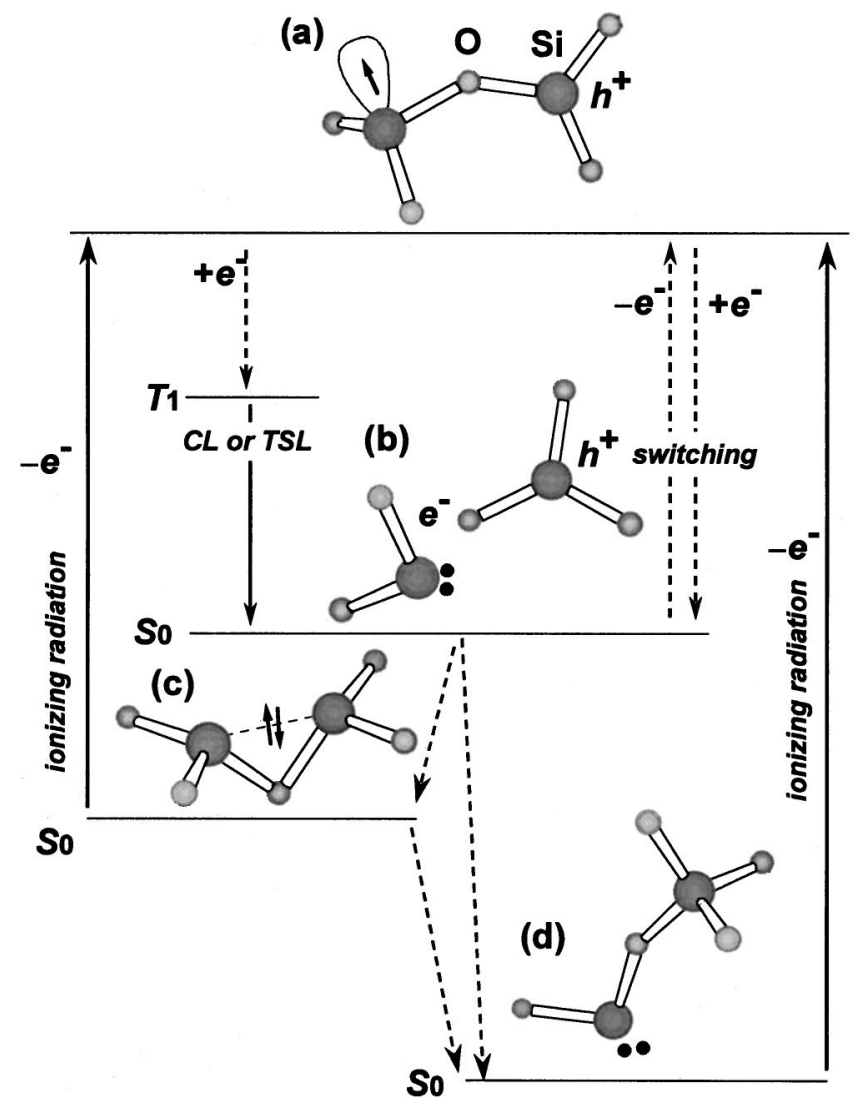

FIG. 4. Schematic models for a switching oxide trap and its related emissions, thermal relaxation and ionizing radiation processes: (a) the bridged hole-trapping oxygen-deficiency center (model 1) that can capture an electron; (b) the structural complex (model 2) that can emit an electron. This complex consists of a hole-electron pair and a divalent defect. The assumed energy level for the first excited triplet state $\left(T_{1}\right)$, which is responsible for cathodoluminescence (CL) and thermally stimulated luminescence (TSL) emissions, is also shown for convenience; (c) the triangular oxygen-deficiency center (model 3); (d) the divalent Si defect (model 4). Recombination and/or thermal relaxation steps are indicated by broken lines.

cesses it is quite likely that the released electron first trapped at BHODC moves to the triplet state of the divalentlike center in model 2, and further relaxation of the triplet state to a lower-lying singlet state may give rise to prompt recombination luminescence at $445 \mathrm{~nm}$ as in the case of the $445-\mathrm{nm}$ photoluminescence induced from photoexcitation of the usual divalent defect. Thus, the present electron trapping model of BHODC will give a reasonable explanation for the 445-nm CL and TSL emissions from $a-\mathrm{SiO}_{2}$.

Furthermore, it has been found that model 2 is a metastable structure, relaxing into more stable configurations as shown in Fig. 3. In this work, we found two configurations whose total energies are lower than that of model 2: one is shown in Fig. 3(a) (model 3), and the other in Fig. 3(b) (model 4). Models 3 and 4 have been found to be lower in total energy than model 2 by 0.05 and $1.23 \mathrm{eV}$, respectively, at the B3LYP/6-31G(d) level. The configuration of model 3 is called TODC as mentioned earlier. In model 3 , the Coulomb interaction between $\mathrm{Si} 2$ and $\mathrm{O} 6$ that can be seen in 
model 2 does not exist $\left(d_{\mathrm{Si} 2-\mathrm{O} 6}=4.611 \AA\right)$, indicating that the transformation from model 2 to model 3 must be accompanied by breaking of this Coulomb interatomic interaction. On the other hand, the configuration of the defect center in model 4 can be viewed as a usual divalent $\mathrm{Si}$ defect. The formation of model 4 will be accomplished by forming the $\mathrm{Si} 1-\mathrm{O} 1$ and $\mathrm{Si} 2-\mathrm{O} 2$ bonds at the expense of the $\mathrm{Si} 1-\mathrm{O} 2$ bond in model 2. The present model of hierarchical relaxation is illustrated in Fig. 4. Thus, the transformation from model 2 to models 3 and 4 will require atomic rearrangements not only in the defect site of interest but in its more remote coordination spheres (i.e., second- and/or thirdcoordination shells). It should hence be worth mentioning that the defect shown in model 2 will not spontaneously transform into lower energy configurations (models 3 and 4); that is, this transformation is possible only after overcoming a considerable barrier associated with bond breaking and its subsequent atomic rearrangements in the $\mathrm{SiO}_{2}$ network. Unfortunately, we have not evaluated the barrier since the barrier position should be obtained using a sophisticated saddlepoint calculation, which is indeed a challenging task for such large clusters. Nevertheless, we still believe that the barrier to reconstruction from model 2 to models 3 and/or 4 will be far higher than that evaluated from the conventional $\mathbf{E}_{\gamma}^{\prime}$ cen- ter model $(\sim 0.2 \mathrm{eV})$ because the former process requires large-scale cooperative atomic rearrangements around the defect.

In conclusion, we have developed a model for the switching and annealing behavior of several defect centers in $a-\mathrm{SiO}_{2}$, shown in Fig. 4, in which BHODC can switch charge state reversibly without inducing complex structural rearrangements. It is hence probable that BHODC, which is an alternative model of the $\mathbf{E}_{\gamma}^{\prime}$ center proposed recently, behaves as a switching oxide trap. The present model also explains the reason why the $\mathbf{E}_{\gamma}^{\prime}$ center yields the $445-\mathrm{nm} \mathrm{CL}$ and TSL emissions, in agreement with the triplet-to-singlet photoluminescence band due to the divalent Si defect. Thus, we can conclude that the present model of hierarchical relaxation associated with BHODCs reasonably accounts for the charge trapping behavior along with its related microscopic structural changes in $a-\mathrm{SiO}_{2}$.

We would like to thank the Supercomputer Laboratory, Institute for Chemical Research, Kyoto University, for providing the computer time. This work was supported in part by Grant-in-Aids for COE Research on Elements Science (12CE2005) form the Ministry of Education, Science, Sports and Culture, Japan.
*Email address: uchino@scl.kyoto-u.ac.jp

${ }^{1}$ J. R. Schwank et al., IEEE Trans. Nucl. Sci. 31, 1434 (1984).

${ }^{2}$ A. J. Lelis, H. E. Boesch, Jr., T. R. Oldham, and F. B. McLean, IEEE Trans. Nucl. Sci. 35, 1186 (1988).

${ }^{3}$ L. P. Trombetta, G. J. Gerardi, D. J. DiMaria, and E. Tierney, J. Appl. Phys. 64, 2434 (1988).

${ }^{4}$ R. K. Freitag, D. B. Brown, and C. M. Dozier, IEEE Trans. Nucl. Sci. 41, 1828 (1994).

${ }^{5}$ See, for example, E. H. Nicollian and J. R. Brews, MOS (Metal Oxide Semiconductor) Physics and Technology (John Wiley \& Sons, New York, 1982).

${ }^{6}$ J. F. Conley, Jr., P. M. Lenahan, A. J. Lelis, and T. R. Oldham, Appl. Phys. Lett. 67, 2179 (1995).

${ }^{7}$ A. J. Lelis, T. R. Oldham, H. E. Boesch, Jr., and F. B. McLean, IEEE Trans. Nucl. Sci. 36, 1808 (1989).

${ }^{8}$ A. H. Edwards, W. B. Fowler, and J. Robertson, in Structure and Imperfections in Amorphous and Crystalline Silicon Dioxide, edited by R. A. B. Devine, J-P. Duraud, and E. Dooryhée (John
Wiley \& Sons, Chichester, 2000), p. 253 and references therein. ${ }^{9}$ K. C. Snyder and W. B. Fowler, Phys. Rev. B 48, 13238 (1993).

${ }^{10}$ M. Boero, A. Pasquarello, J. Sarnthein, and R. Car, Phys. Rev. Lett. 78, 887 (1997).

${ }^{11}$ P. E. Blöchl, Phys. Rev. B 62, 6158 (2000).

${ }^{12}$ F. J. Feigl, W. B. Fowler, and K. L. Yip, Solid State Commun. 14, 225 (1974).

${ }^{13}$ T. Uchino, M. Takahashi, and T. Yoko, Phys. Rev. B 62, 2983 (2000); Phys. Rev. Lett. 86, 5522 (2001).

${ }^{14}$ B. B. Stefanov et al., Phys. Rev. Lett. 81, 3908 (1998).

${ }^{15}$ M. S. Gordon, Chem. Phys. Lett. 76, 163 (1980), and references therein.

${ }^{16}$ C. Lee, W. Yang, and R. G. Parr, Phys. Rev. B 37, 785 (1988).

${ }^{17}$ A. D. Becke, J. Chem. Phys. 88, 1053 (1988).

${ }^{18}$ G. R. Atkins et al., J. Lightwave Technol. 11, 1973 (1993).

${ }^{19}$ M. Martini et al., J. Non-Cryst. Solids 187, 124 (1995).

${ }^{20}$ L. Skuja, J. Non-Cryst. Solids 167, 229 (1994). 